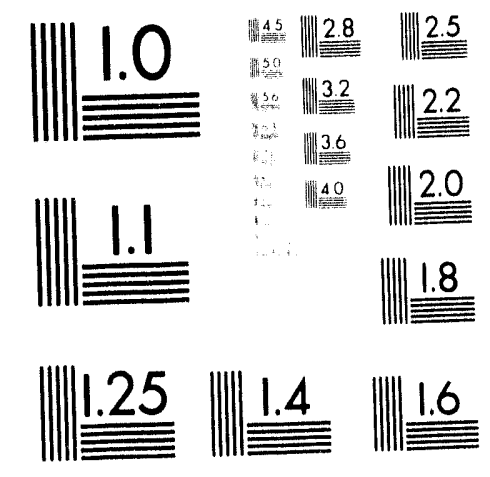



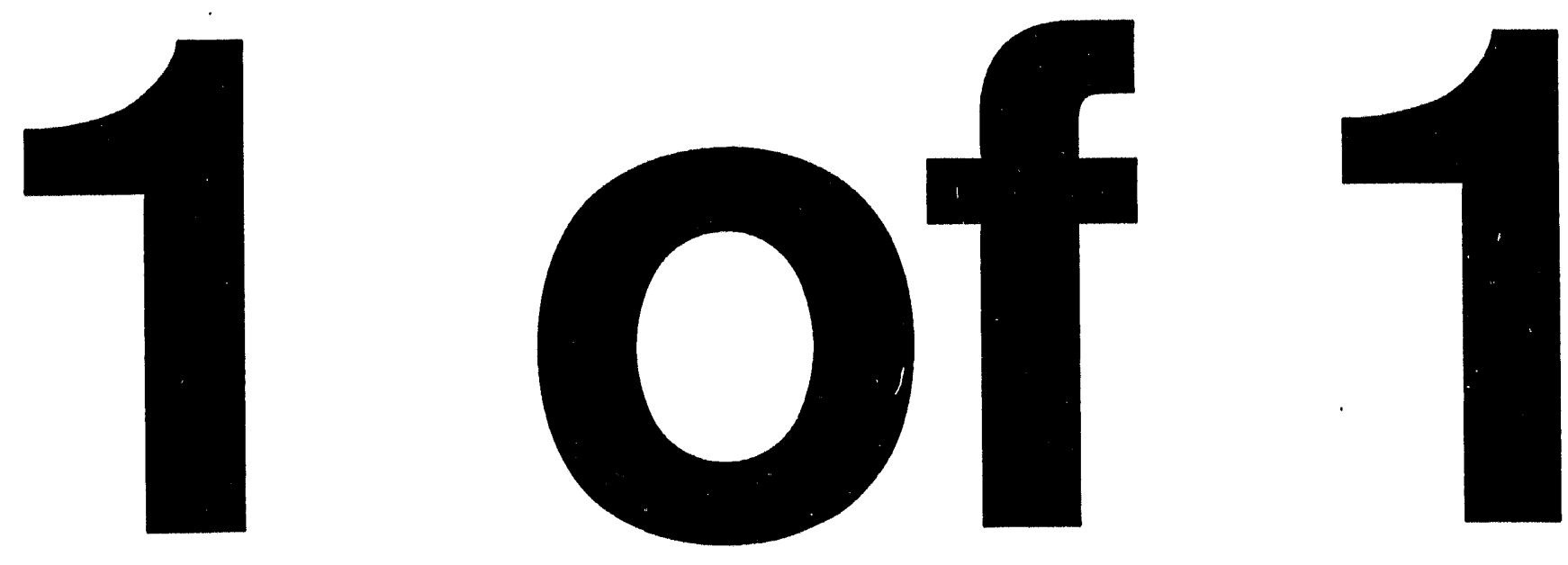
LA-UR- $93-3504$
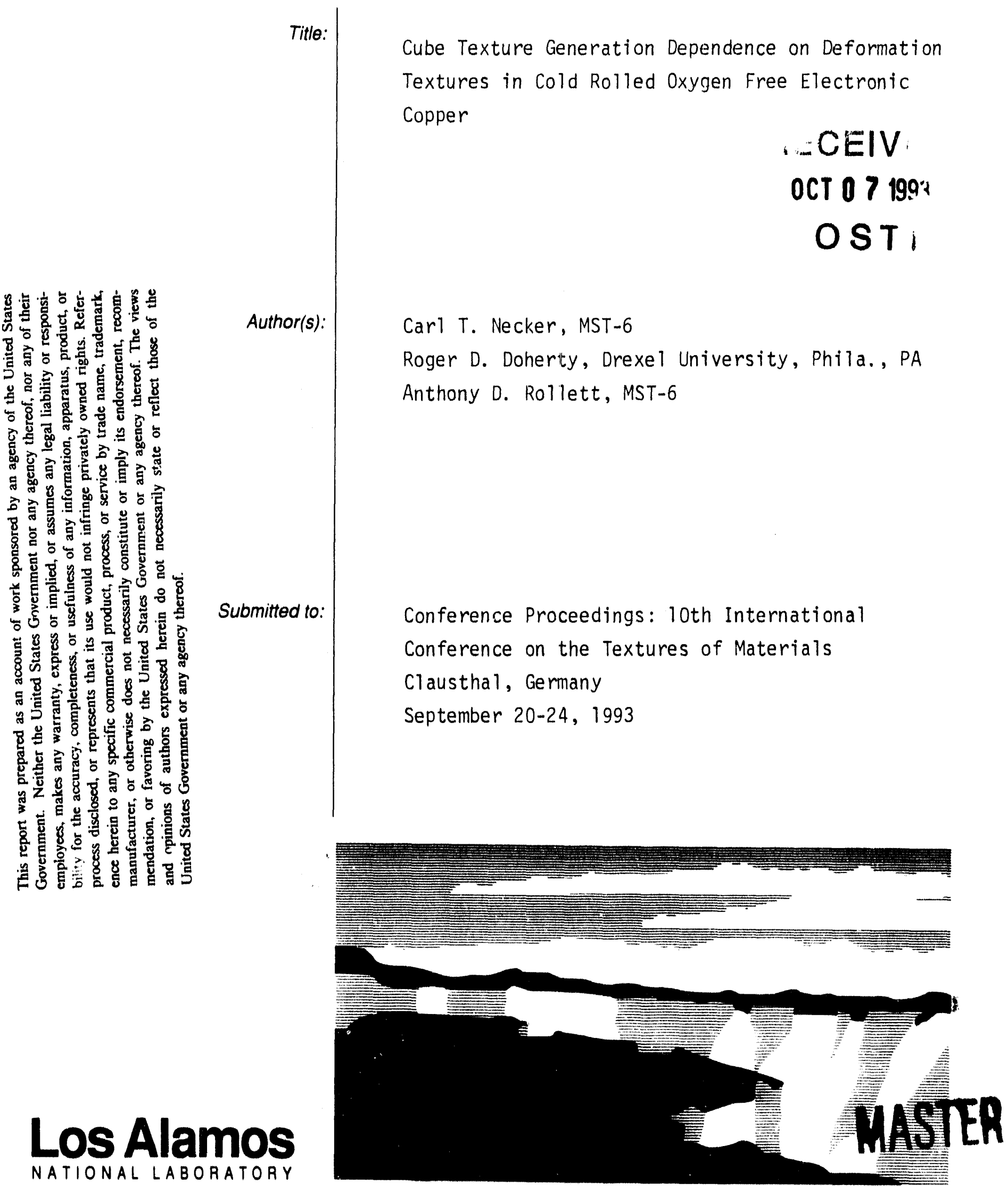

NATIONAL LABORATORY

Los Alamos National Laboratory, an atfirmative actionvequal opportunity employer, is operated by the University of Callfornia tor the U.S. Department of Energy under contract W.7405-ENG-36. By acceptance of this article, the publisher recognizes that the U.S. Government retains a nonexclusive, royaity-tree license to publish or reproduce the published form of this contribution, or to allow others to do so, for U.S. Governmemt purposes. The Los Alamos National Laboratory requests that the publisher identify this article as work performed under the auspices of the U.S. Department of Energy.

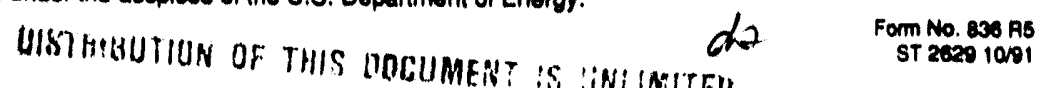




\title{
CUBE TEXTURE GENERATION DEPENDENCE ON DEFORMATION TEXTURES IN COLD ROLLED OFE COPPER
}

\author{
C. T. Necker* , R. D. Doherty ${ }^{+}$, A. D. Rollett ${ }^{*}$ \\ *Los Alamos National Laboratory, Los Alamos, New Mexico 87545 \\ +Drexel University, Philadelphia, Pennsylvania 19104
}

Key Words: Cube Texture, $\beta$ Fiber, Nucleation, Growth, Recrystallization

\section{ABSTRACT}

The evolution of the $\alpha$ and $\beta$ fiber deformation textures in OFE copper for von Mises strains of 1.0 to 4.5 is reported as well as how it affects the generation of cube recrystallization textures. Computational volume fraction analysis of the fibers indicates that the deformation texture evolves evenly along the length of the fibers. Fiber weakening during recrystallization does not occur more selectively in any one component or section of the fiber but occurs relatively evenly across the fiber. Cube grains grow without misorientation preference into the deformed structure. Microtextural analysis of the fully recrystallized samples indicates that the cube component (defined as less than $20^{\circ}$ misorientation from $\{100\}<001>$ ) not only strengthens with increasing prior strain but also becomes much sharper, more closely aligned with the exact cube position. These results are explained by the evolution of the environment (texture and microstructure) surrounding potential nucleation sites.

\section{INTRODUCTION}

The origin of cube recrystallization textures in f.c.c. metals has long been the object of research and discussion. The cold rolling deformation textures and microstructures that yield cube recrystallization textures upon annealing have been extensively studied, however, the actual transition, that is, the decay of deformation textures during recrystallization has not (1-4). It is well known that heavily cold rolled copper will produce a partial fiber texture, most often identified as the $\alpha$ and $\beta$ fibers. These are defined as "tubes" of intensities that connect the deformation texture components: brass, $\{011\}<211>$, to Goss, $\{011\}<100>$ and brass through $S,(123\}<634>$, to copper, $(112\}<111>$. The fibers and components are often quantitatively analyzed as a way of defining the development of the rolling texture. However the entire fiber, not just the components, should be analyzed during recrystallization because the entire fiber is almost completely consumed by the cube oriented grains, $\{001\}<100\rangle$, during recrystallization of heavily rolled copper. The primary questions that arise concerning the origin of cube textures are, do cube nuclei consume particular orientations within the fiber, and, do cube nuclei have particular misorientations with their neighboring deformed material?

In an effort to determine if particular orientations are preferred as a growth environment for cube orientations, the deformation textures for different levels of cold rolling have been characterized by determining volume fractions for $\alpha$ and $\beta$ fibers as well as for components. One particular rolling reduction, $90 \%$, was chosen to study the decay of the fibers and components during recrystallization. Additionally, microtexture analysis was employed to further define the cube recrystallization state. Three levels of cold rolling, $73 \%, 90 \%$ and $98 \%$ (von Mises strains of 1.5, 2.5 and 4.5), were studied 
in the fully recrystallized state to examine the misorientations between a perfectly oriented cube grain and 'cube' grains within the samples, defined as misorientations within $20^{\circ}$ of perfect cube. This technique allows for the determination of numbers of recrystallized grains with particular orientations, sizin of grains as a function of orientation as well as the calculation of misorientations. These factors are useful in the characterization of the cube recrystallization environment which in turn may be used to determine whether preferential formation of cube textures is dependent on nucleation or growth phenomena, or both.

\section{EXPERIMENTAL PROCEDURE}

Oxygen free electronic (OFE) copper, containing $10 \mathrm{ppm}$ oxygen as the primary impurity and having a nearly random texture, was cold rolled to six levels of reduction between $58 \%$ and $98 \%$ (von Mises strains of 1.0 to 4.5 , respectively). Samples from each rolling reduction were partially recrystallized in a silicone oil bath, yielding a series of texture samples that range from deformed to fully recrystallized. The sample rolling plane, half depth surfaces were metallographically prepared (ground, polished, etched) for texture analysis on a Scintag 5 Axis pole figure goniometer. The data was analyzed using popLA (Preferred Orientation Package Los Alamos) software to calculate the orientation distributions. The volume fractions of the $\alpha$ and $\beta$ fibers as well as various deformation (copper, S, brass, Goss) and recrystallization (cube and twin of cube, $\{122\}<212>$ ) texture components were determined by recalculating the orientation distributions based on a correction for Euler volumes. These analyses were performed to study the development of the rolling deformation texture and the transformation from rolled to recrystallized state. Following the texture measurements, the rolled samples were mounted in epoxy and metallographically prepared on the long transverse surface (the plane containing the rolling plane normal and rolling directions), for quantitative microscopy and orientation imaging microscopy (microtexture analysis). Tell photographs per sample, at 400 magnification, were taken on a CamScan Series 4 scanning electron microscope in backscatter mode and were statistically analyzed to determine the volume fraction recrystallized. The local texture of recrystallized regions of several fully recrystallized samples was measured using a Jeol 840 SEM equipped with a low level light camera and analysis system driven by Dingley software (5).

\section{RESULTS AND DISCUSSION}

The development of $\beta$ and $\alpha$ fibers during the cold rolling of OFE copper is presented in Figures $1 \mathrm{a}$ and $1 \mathrm{~b}$. The development was characterized by the volume fraction of the fibers which in turn are defined as a series of maxima along the fiber length and the spread of intensity around those maxima. See Figure 2 for an example of a plot of the orientation distribution showing the fibers. In this way the fiber is effectively sliced into constant angle increment cross sections in $\psi$ angle increments with the volume fraction being calculated by a simple summation of these increments, each defined by angular spreads in $\Theta$ and $\Phi$ about each maxima ${ }^{+}$. See Equations 1 and 2 .

$$
\begin{gathered}
\text { Euler Volume }=\int \sin \Theta \mathrm{d} \Theta \mathrm{d} \Phi \mathrm{d} \psi \\
\text { Volume Fraction }=\left(\Sigma\left(\left(\cos \Theta_{1}-\cos \Theta_{2}\right) * D\right)\right) / V_{\mathrm{T}}
\end{gathered}
$$

where $D$ is the orientation distribution density at a given angular position and $V_{T}$ is the total volume of the texture, calculated in the same way as the numerator of equation 2 . The same calculations were performed to determine the volume fraction of the fibers had the texture actually been random. Within the fibers certain slices are defined as particular component positions, brass (Bs), S, copper $\mathrm{Cu}$ and Goss. However, the analysis of components avoids the use of ideal orientation positions because the fiber is not fixed during deformation. Hirsch's work shows that the fiber maxima move

\footnotetext{
+ Angles follow Kocks' convention for Euler angles (6).
} 
during rolling, by as much as five degrees, making it difficult to label "ideal" orientations because of the uncertainty in their positions (1). For the current analysis, the component positions were placed within particular $\psi$ sections.

Figure 1a shows the change in volume fraction in one section of the $\beta$ fiber, the transition from $\mathrm{Bs}$ to $\mathrm{S}$ to $\mathrm{Cu}$. The volumetric strengthening of the fiber is not dominated by any one component or section of the fiber but occurs relatively evenly across the fiber. A similar analysis of the other two sections of the $\beta$ fiber, related by symmetry elements, exhibits the same trend. The volume fractions are small because they represent only one of the three symmetrically equivalent parts of the fiber. By taking into account the entire fiber, the $\beta$ fiber represents approximately $50 \%$ of the entire deformation texture after 58\% rolling and $70 \%$ of the texture after $95 \%$ rolling. Other similar analyses typically show a volumetric strengthening along the $\beta$ fiber with a peak forming around the $S$ component, close to $\psi=60^{\circ}$, which develops between the $85 \%$ and $95 \%$ rolling reductions (1-3). The current volume analysis yields the same general trend if slices are added together from each side of the $S$ component. The $\alpha$ fiber, Figure $1 b$, is fully developed at the earliest rolling reductions investigated. With the exception of the minor strengthening of the Bs end of the fiber, the volume of the fiber changes little, varying between $15 \%$ and $18 \%$ of the entire deformation texture. After $95 \%$ reduction, approximately $85 \%$ of the texture is within the $\alpha$ and $\beta$ fibers, the remainder being randomly scattered intensities.

The same analysis as performed above was used to look at the transformation between deformation to recrystallization textures in $90 \%$ rolled copper. Figures $3 \mathrm{a}$ and $3 \mathrm{~b}$ show the decay of the same sections of the $\beta$ and $\alpha$ fibers as recrystallization progressed. Decay is defined as a combination of weakening of intensities and the narrowing of the spread around a local position. No one section of the $\beta$ fiber appears to decay faster than another. The minor peak in Figure 3a around $\psi=65^{\circ}$ in the $92 \%$ recrystallized data is due to the proximity of the cube twin component to the $\beta$ fiber. If the analytical spread in this section of the fiber, typically $10^{\circ}-15^{\circ}$ for both $\Theta$ and $\Phi$, were narrower then the peak would disappear. The $\alpha$ fiber decays preferentially where the $\alpha$ and $\beta$ fibers connect, around $\psi=60^{\circ}$, at the Bs component. These results, coupled with the non-linear strengthening of the cube recrystallization component, Figure 4, mean that cube grains growing into deformed material do not preferentially grow at the expense of certain parts of the $\beta$ fiber. If this were not the case, i.e. cube grains grow only into material with certain misorientations, then the fiber would not be expected to decay evenly.

It does appears that the $\beta$ fiber is preferentially consumed rather than the $\alpha$ fiber. Additionally, at the $95 \%$ and $98 \%$ reduction levels the recrystallization texture is almost completely cube and twin of cube therefore the fiber must be consumed almost exclusively by cube oriented grains, irrespective of misorientation. From a macroscopic texture perspective, it appears unlikely that cube grains need a preferential misorientation environment to grow. From a microscopic texture perspective, cube nuclei may need a preferential misorientation in order to form a grain, e.g. $\left(25^{\circ}\right.$ $4\left(0^{\circ}\right)<111>(7)$. Once the nucleus begins to grow then it may be indifferent as to what it grows into. It will continue to grow until it a) impinges upon another recrystallized grain, b) the misorientation becomes insufficient to allow the boundary to remain mobile, or c) solute/impurities build up on the boundary stopping migration (8). The scale of initial growth misorientation preference may be too small to measure using $X$-ray diffraction techniques.

The microtexture analysis of fully recrystallized samples shows that recrystallized cube grains increase in number per unit volume and become more closely aligned to the perfect cube orientation as a function of increased prior strain. Approximately 5\% of the orientations measured in the $73 \%$ rolled, fully recrystallized material were classified as cube. The number of cube orientations grew to $30 \%$ in the $90 \%$ rolled material and to about $95 \%$ in the $98 \%$ rolled material. There are two possible explanations for the increased frequency of cube grains as well as the evolution of cube grain orientations. During deformation, material could rotate toward the cube orientation and then 
nucleate. The second option is that material with small misorientations from cube, $\left(0^{\circ}-15^{\circ}\right.$, could have already existed in the deformed material. The surrounding material would then evolve into an environment conducive for growth - sufficient misorientations, local stored energy, etc. Based on work by Dillamore and Katoh, it is possible that under certain circumstances (fixed constraints deformation theory) material rotated initially only about the cube rolling plane normal direction could rotate back to the cube orientation during deformation (9). However, under the conditions of large rolling reductions it is unlikely that this could account for the sharpening of the cube texture. Dillamore and Katoh's work on cube centered transition bands does allow for a more viable explanation. We speculate that the environment local to a band could evolve such that at $90 \%$ reduction the material misoriented from cube by $10^{\circ}-15^{\circ}$ is next to material with an environment conducive for nucleation and growth. By $98 \%$ reduction this same type of environment has developed next to material more closely oriented to cube. These cube sites have such an advantage over other sites that virtually all active nucleation sites must have been cube. Rolled copper could therefore develop the local environment necessary to produce a range of recrystallization textures from a random level of cube to a virtually single crystal texture, as found in the $98 \%$ rolled and recrystallized copper. The percentage of cube grains within a given misorientation are presented below as a function of prior rolling reduction:

\section{TABLEL}

\section{Misorientation from Cube (degrees) ${ }^{*}$}

$$
\begin{gathered}
0-5 \\
5-10 \\
10-15 \\
15-20
\end{gathered}
$$

$\begin{array}{ccc}73 \% \text { rolled } & \text { 20\% rolled } & \text { 28\% rolled } \\ 0 \% & 0 \% & 60 \% \\ 10 \% & 5 \% & 30 \% \\ 30 \% & 85 \% & 10 \% \\ 60 \% & 10 \% & 0 \%\end{array}$

\section{CONCLUSIONS}

Cold rolling OFE copper from $58 \%$ to $98 \%$ reductions strengthens the $\beta$ fiber evenly along its length. Although maxima in certain regions of the fiber increase more rapidly, this intensification is offset by the spreading of the fiber - a more intense skeletal line in a broader fiber. These results corroborate the work of others. The decay of the deformation texture occurs relatively evenly along the length of the $\beta$ fiber. This result coupled with the non-linear intensification of the cube recrystallization texture means cube grains grow into their neighbors, irrespective of misorientation, as long as the misorientation is sufficient for the boundary to be mobile. Cube oriented material exists at all rolling reductions studied, however, it does not gain its recrystallization advantage until the $82 \%$ reduction, yielding cube recrystallization textures that strengthen steadily from $2-3 \times$ random to $150 \mathrm{x}$ random at the highest reduction, $98 \%$. The microtexture analysis results show a sharpening of the recrystallization cube orientations and an increased frequency in the number of cube grains. The strengthening and transition of cube orientations may be explained by the development of the deformation environment around the cube orientations in deformed material. Deformation causes a constant evolution of nucleation sites and the environment around these sites. There were many noncube nucleation sites in the $90 \%$ rolled material but virtually none in the $98 \%$ rolled copper meaning there is a preferential nucleation of cube grains. The increased frequency also indicates that the strengthening of the cube texture is not purely a growth phenomena.

\footnotetext{
The $73 \%$ and $90 \%$ rolled and recrystallized samples produced many annealing twins, often making it difficult to record only one orientations per grain (ex. two cube orientations separated by a twin orientation or another orientation could be registered as a single grain or as two). The above values are estimated to be skewed by $+1.10 \%$. This problem did not exist in the $98 \%$ rolled and recrystallized material since the only recrystallization texture components were cube and twin of cube, the twins being orders of magnitude smaller than the cube grains. One orientation per grain wa; recorded.
} 


\section{ACKNOWLEDGMENTS}

The authors thank ALCOA (Aluminum Company of America) and Dr. John Liu for the use of their microtexture equipment. Discussions with U. F. Kocks and A. J. Beaudoin were also instrumental in the development of the volume analysis program. The work was supported by National Science Foundation Grant DMR 9001378 and a Department of Energy contract to Los Alamos National Laboratory, W-7405-ENG-36.

\section{REFERENCES}

1. J. Hirsch, Ph.D. Thesis, RWTH Aachen, 1984.

2. K. Lücke, J. Pospiech, J. Jura and J. Hirsch, Z. Metallkde, 77 (1986), 312.

3. H. Hammelrath, J. F. Butler, H. Hu and K. Lücke, "Deformation and Recrystallization Textures of Rolled and Channel-Die Compressed High Purity Copper", ICOTOM-9, (Avignon, France, 1990), 629.

4. C. T. Necker, R. D. Doherty and A. D. Rollett, "Quantitative Measurement of the Development of Recrystallization Texture in OFE Copper", ICOTOM-2, (Avignon, France, 1990), 635.

5. D. J. Dingley, "On Line Microtexture Determination Using Backscatter Kikuchi Diffraction ina Scanning Electron Microscope", ICOTOM-8, (Santa Fe, New Mexico, 1988), 189.

6. U. F. Kocks, "A symmetric Set of Euler Angles and Oblique Orientation Space Sections", ICOTOM-8, (Santa Fe, New Mexico, 1988), 31.

7. $\quad$ B. J. Duggan, K, Lucke, G. Köhlhoff and C. S. Lee, Acta Met., 41 (1993), 1921.

8. F. Haessner and S. Hofmann, "Migration of High Angle Grain Boundaries", Recrystallization of Metallic Materials, Ed. F. Haessner, (Stuttgart, Technische Universităt Braunschweig, 1978), 63.

9. I. L. Dillmore and H. Katoh, Metal Sci, 8 (1974), 73.

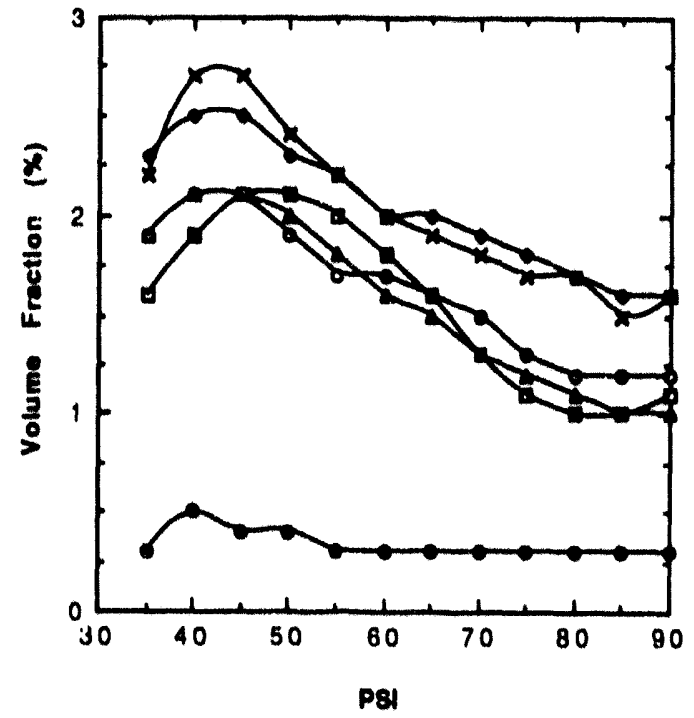

Figure 1a. Beta fiber evolution during rolling.

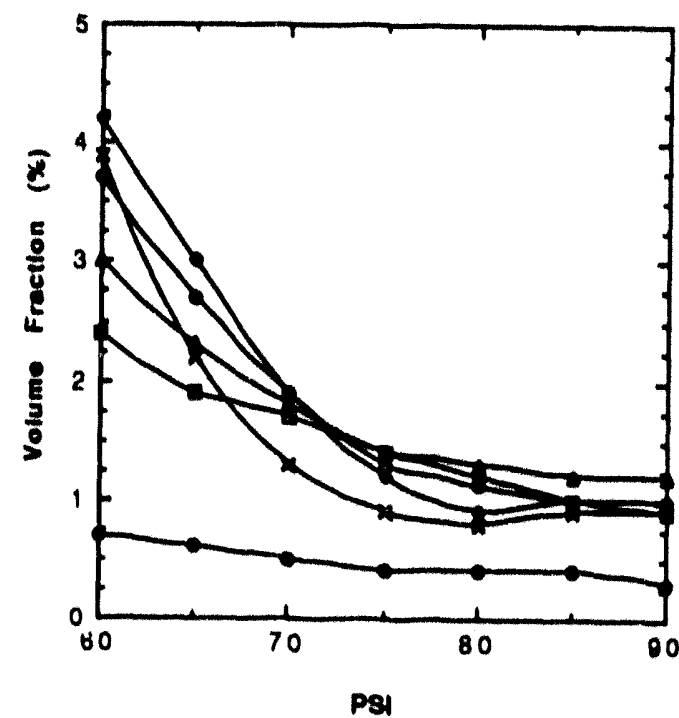

Figure Ib. Alpha fiber evolution during rolling. 

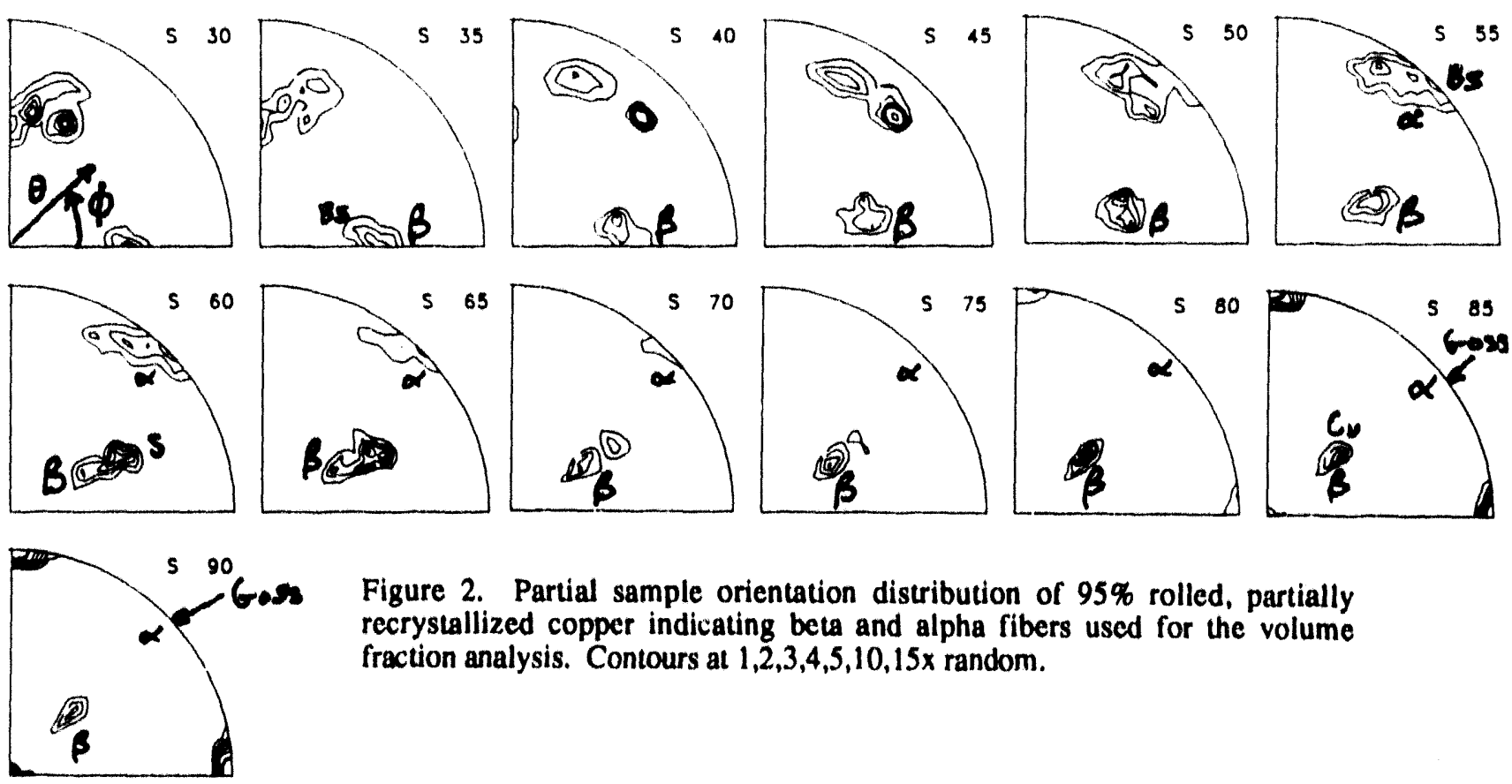

Figure 2. Partial sample orientation distribution of $95 \%$ rolled, partially recrystallized copper indicating beta and alpha fibers used for the volume fraction analysis. Contours at $1,2,3,4,5,10,15 \times$ random.

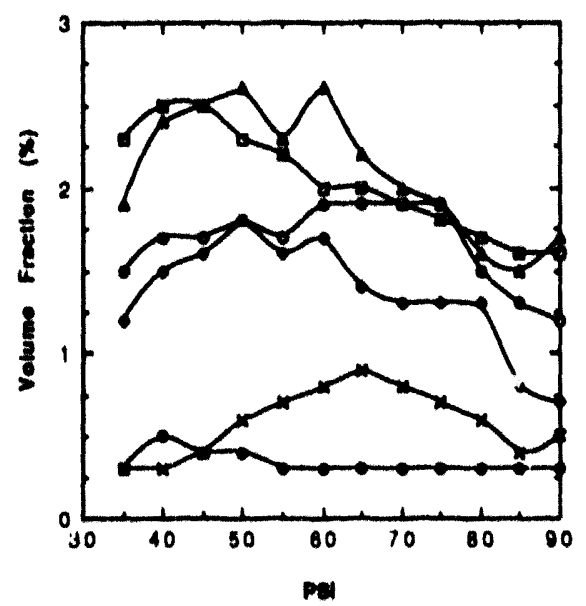

Figure 32. Beta fiber docay during recrystallization.

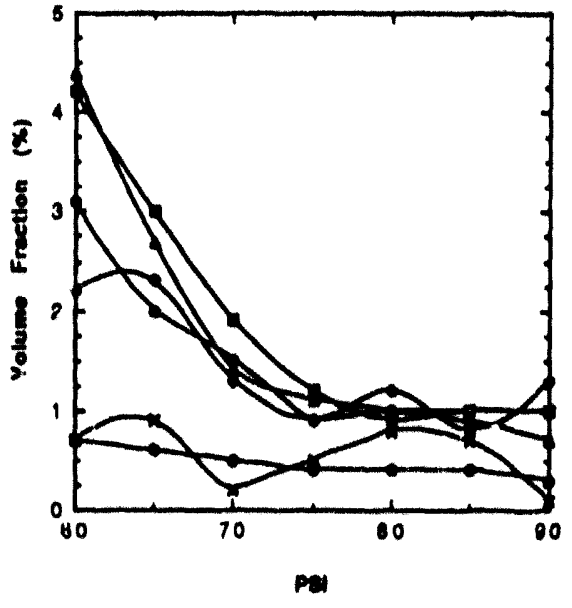

Figure 3b. Alpha fiber decay during recrystullization.

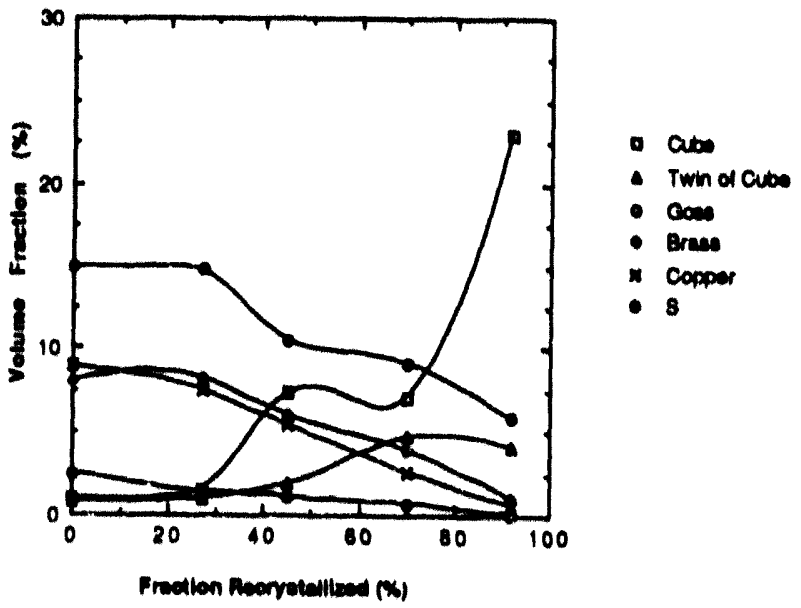

Figure 4. Evolution of components during recrystallization. 

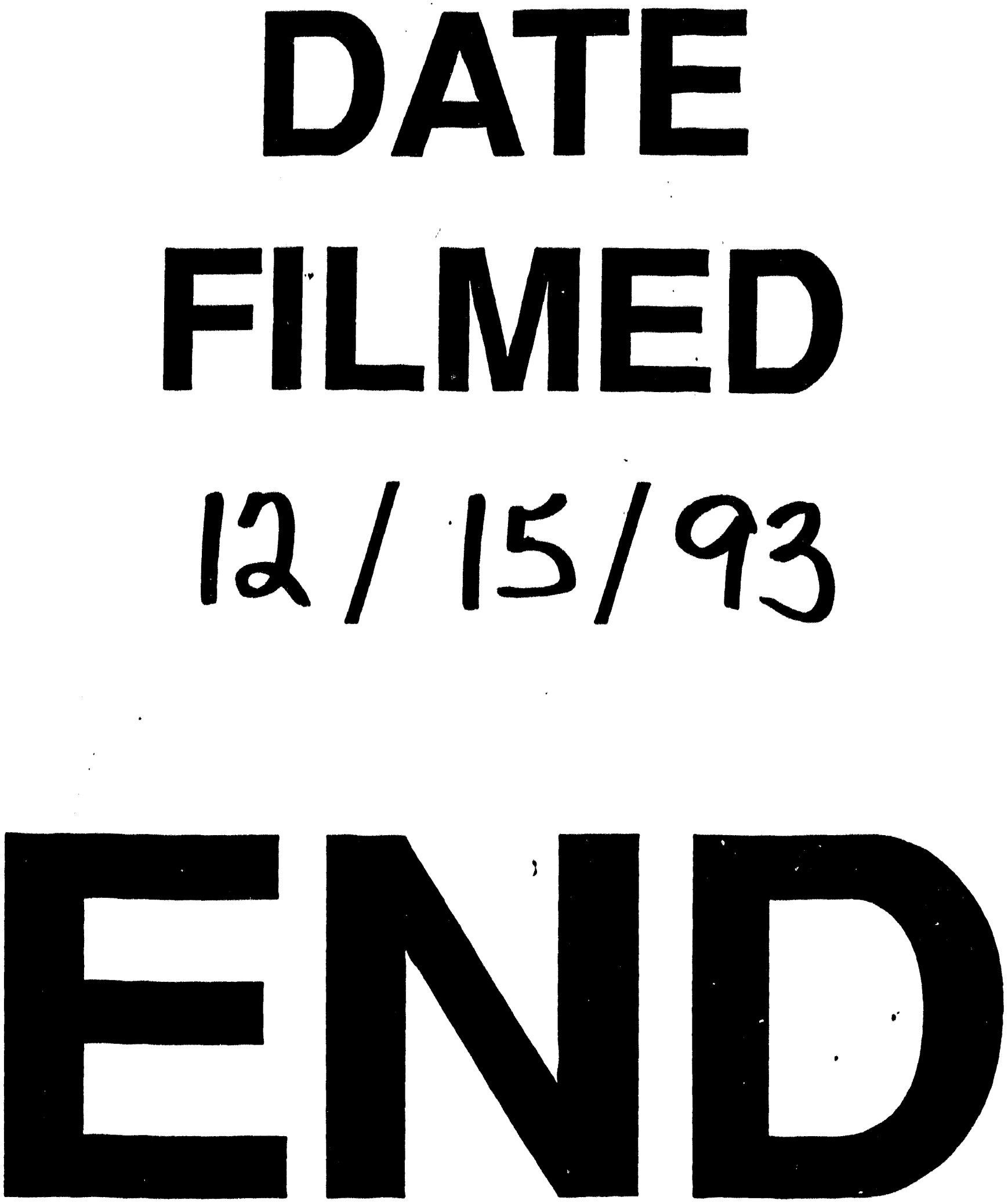


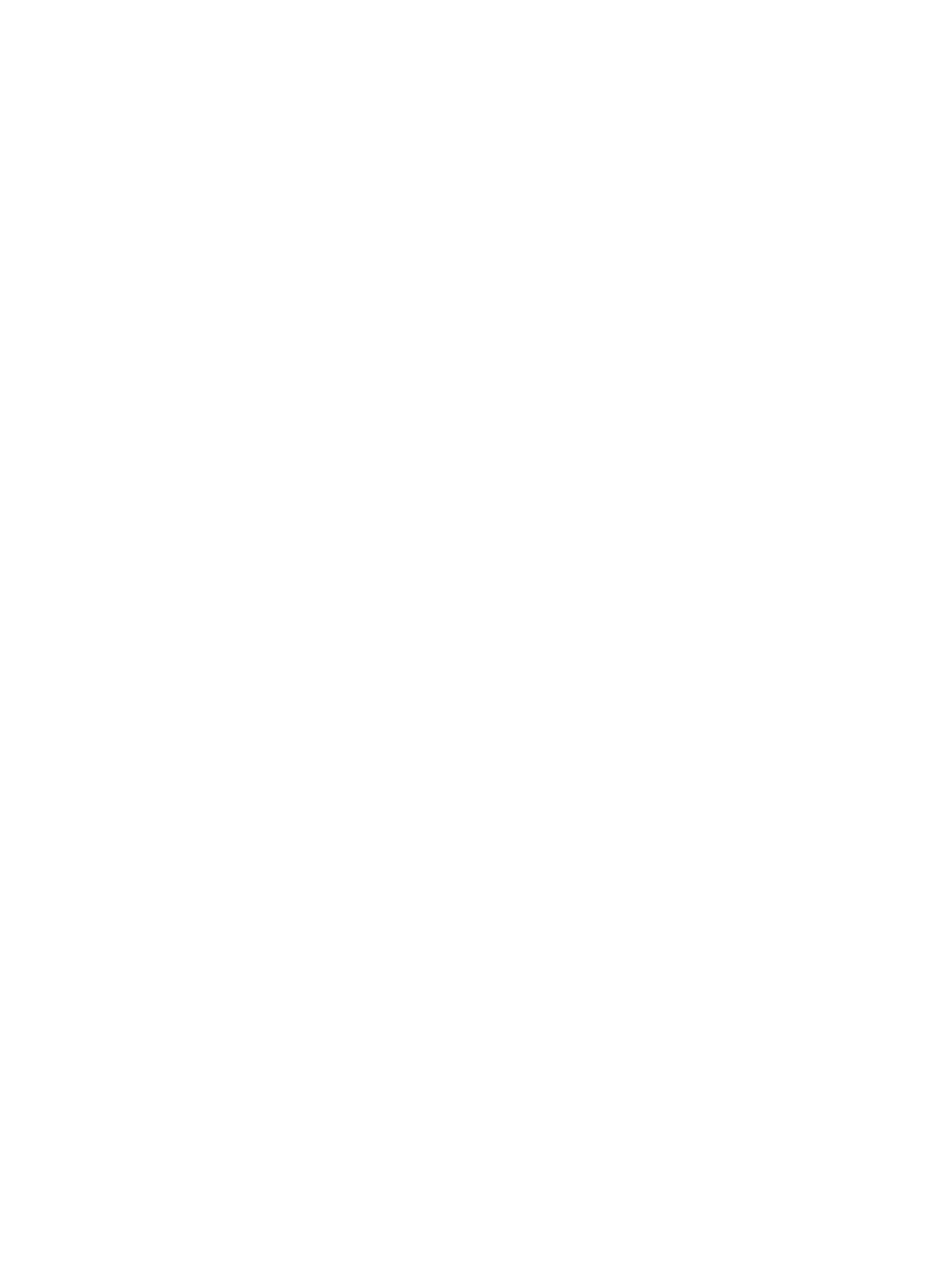

